\title{
Correlation Between Contrast-enhanced Ultrasound Characteristics (Qualitative and Quantitative) and Pathological Prognostic Factors in Breast Cancer
}

\author{
IRENE VRAKA ${ }^{1}$, EVANGELIA PANOURGIAS $^{1 *}$, EMMANOUIL SIFAKIS $^{2 *}$, ANDREAS KOUREAS $^{1^{* *}}$, \\ PETROS GALANIS $^{3 * *}$, DIONYSIOS DELLAPORTAS ${ }^{4}$, ATHANASIOS GOULIAMOS ${ }^{1}$ and ARISTIDES ANTONIOU ${ }^{1}$ \\ ${ }^{1}$ Department of Radiology, Aretaieion Hospital, Department of Medicine, \\ National \& Kapodistrian University of Athens, Athens, Greece; \\ ${ }^{2}$ Department of Oncology-Pathology, Jonas Bergh Research Group, Karolinska Institute, Stockholm, Sweden; \\ ${ }^{3}$ Center for Health Services Management and Evaluation, Department of Nursing, \\ National \& Kapodistrian University of Athens, Athens, Greece; \\ ${ }^{4} 2$ nd Department of Surgery, Aretaieion Hospital, Department of Medicine, \\ National \& Kapodistrian University of Athens, Athens, Greece
}

\begin{abstract}
Background/Aim: Little is known about the correlation between contrast-enhanced ultrasound (CEUS) characteristics and pathological prognostic factors in breast cancer. The aim of this study was to explore the correlation between CEUS characteristics and pathological prognostic factors. Patients and Methods: A retrospective study with 34 malignant breast lesions was conducted. CEUS characteristics included qualitative characteristics (e.g. lesion's enhancement degree and order, internal lesion homogeneity etc.) and quantitative characteristics (e.g. peak intensity, time to peak etc.). Also, pathological prognostic factors were included (e.g. tumor grade, estrogen receptor status etc.). Results: Blurred lesion margins were observed more often in tumors of high histological grade $(p=0.01)$ and in estrogen receptor-negative tumors ( $p=0.049)$. Furthermore, perilesional enhancement was associated with positive Ki-67 expression $(p=0.049)$, while heterogeneous internal sentinel lymph node enhancement was associated with malignant infiltration of the node $(p=0.002)$.
\end{abstract}

This article is freely accessible online.

*E. Panourgias and E. Sifakis contributed equally to this study. **A. Koureas and P. Galanis contributed equally to this study.

Correspondence to: Petros Galanis, MPH, Ph.D., Center for Health Services Management and Evaluation, Department of Nursing, National \& Kapodistrian University of Athens, 123 Papadiamantopoulou St., GR11527, Athens, Greece. Tel: +30 2107461471, Fax: +30 2107461491, e-mail: pegalan@nurs.uoa.gr

Key Words: Breast cancer, contrast-enhanced ultrasound, prognosis, qualitative analysis, quantitative analysis.
Conclusion: CEUS has the potential to provide a prevision of pathological prognostic factors in malignant breast lesions, helping in the better early patient management.

Breast cancer is a major public health problem, since worldwide it is the most common cancer in females, with an increased risk of the disease and related mortality $(1,2)$. Furthermore, it is a highly heterogeneous disease and the major risk factors include genetic, life style and environmental factors $(2,3)$.

Tumor size, histologic grade and axillary lymph node status are classic pathological prognostic factors of breast cancer and have been used to predict recurrence and overall survival (4). However, introduction of new treatment managements has revealed an emerging need for preoperative information, so as to achieve the optimal outcome with treatment along with minimum side effects. It is well known that expression of hormone receptors, such as estrogen receptors (ER) and progesterone receptors (PR) guide hormonal therapy administration and predict its outcome, with ER-positive and PR-positive patients having better prognosis (5-7). While ER negative tumors do not respond well to this therapy, they are sensitive to chemotherapy (8). In the same way, biomarkers, such as p53, c-erb-B2 and Ki-67 are useful in patients' prognosis and personalization of neo-adjuvant treatment $(6,9$ 11). Namely, Ki-67 is a nuclear antigen of all proliferating cells and its expression reflects active tumor cell proliferation, while it is correlated with tumor's higher histologic grade and the possibility of lymph nodes' metastatic disease $(10,12)$. All the same, c-erb-B2 proto-oncogene's overexpression and amplification is strongly related to tumor's neoplastic transformation, progression and potential to metastasize (6), while anti-oncogene p53 is subject to mutations in tumors (10). 
Unfortunately, for the present, histological specimens are necessary in order to diagnose these pathological prognostic factors and biomarkers. Therefore, a non-invasive examination tool, which would provide information highly correlated with these factors and performed prior to surgery, would be very helpful in early management of patients with breast cancer. This can be a new potential application of contrast enhanced ultrasound (CEUS), as developments in the domain of medical equipment and new generations of micro-bubble contrast agent augmented the method's accuracy and its role in indicating angiogenesis on the microcirculation level.

There are few studies in literature exploring correlation between CEUS characteristics and pathological prognostic factors in breast cancer patients during the last six years, indicating a novel research area (13-19). Also, these studies have shown mixed results and have had differences in patients' populations, study design, statistical analysis etc. Indicatively, three studies have investigated only qualitative CEUS characteristics' correlation to pathological prognostic factors $(14,15,18)$, two studies have investigated only quantitative CEUS characteristics' $(13,16)$, and only two studies have investigated both quantitative and qualitative CEUS characteristics' $(17,19)$.

Consequently, this study was performed to further explore the correlation between qualitative and quantitative CEUS characteristics and pathological prognostic factors in breast cancer patients.

\section{Patients and Methods}

Patients. A retrospective study was conducted and 40 consecutive patients with suspicious malignant breast lesions in mammography and/or conventional ultrasound examination were initially recruited (BI-RADS 4-5). No one of them met any of the exclusion criteria concerning contrast agent administration, namely severe cardiopulmonary disease or respiratory syndrome, pregnancy and lactation, as well as hypersensitivity.

Firstly, a contrast enhanced ultrasound examination was performed in all patients and then they underwent breast surgery. Afterwards, a pathological and immunohistochemical analysis of all removed tumors took place. Finally, 32 patients were enrolled in this study, because of lack of available histological correlation after surgery (in one case), benignity of resected lesions (in two cases) or poor image quality in received video clips (due to excessive respiratory movement or patient's movement artifacts) in five cases. A total of 34 malignant breast lesions were studied in these 32 patients.

Patients' age ranged from 37 to 85 years old, the mean age was 60.6 years (standard deviation; 13 ) and the median age was 61 years.

Ultrasound examination procedure. The ultrasound images were obtained by a Logiq 9 Ultrasound System, using the Coded Contrast Imaging Technique and by a 101 linear probe, with a frequency range of 5-12MHz. Low mechanical index (MI 011-017), PO 4\% (Power output) and TAD (TruAgent Detection) Imaging Techniques were applied. The contrast agent used was SonoVue (Bracco,
Courcouronnes, France), which was reconstituted by $25 \mathrm{mg}$ of lyophilized powder added in $5 \mathrm{ml}$ sterile solution. All ultrasound examinations with contrast agent were performed by the same physician, with 10 years of experience in breast ultrasound.

In the beginning of the images' achieving procedure, high resolution gray scale ultrasound examination was performed, in order to identify the breast lesion and the sentinel lymph node in each patient. The selection of the lesion's section which was going to be studied after contrast agent infusion was done during the color Doppler examination and it was the one better depicting the presence of vessels in the lesion. The selected image also included lesion's surrounding tissue, where possible.

The lymph node considered as sentinel was selected by its nearest place to the breast lesion, along with possible suspicious morphologic criteria, including (a) increased thickening and/or lobulation of the cortex, compared to other ipsilateral or contralateral nodes, (b) eccentric lobulation of the hypoechoic cortex, with compression of hilar fat, (c) complete replacement of hilar fat by the hypoechoic cortex and (d) round lymph node shape (20).

A recording of $90 \mathrm{sec}$ followed the bolus intravenous injection of $2.4 \mathrm{ml}$ ultrasound contrast media and of $10 \mathrm{ml}$ sterile solution through a $21 \mathrm{G}$ catheter in antecubital vein. This procedure took place twice, in order to obtain one video clip for the breast lesion and another one for the sentinel lymph node. Recordings started just after intravenous contrast agent administration. In the end, all patients remained under inspection for about 30 minutes after intravenous contrast agent injection, according to the guidelines (21), in order to ensure that no side effects would appear.

Contrast enhanced ultrasound image evaluation procedure. Acquired video clips were analyzed on a qualitative and quantitative basis, a procedure performed by two radiologists of equal experience and good knowledge of the previous literature, while both were blinded to the patients' clinical data. In case of discordance, consensus was achieved after image review by a third radiologist.

Evaluated qualitative characteristics included the following: (a) lesion's enhancement degree compared to the surrounding breast tissue, the moment of peak enhancement, demonstrating hypoenhancement, iso-enhancement or no enhancement (b) internal lesion's homogeneity, characterized homogenous when enhancement was visible and equal to the whole lesion's extent and heterogeneous when unenhanced areas or areas of different enhancement degree appeared after contrast medium administration; (c) presence or absence of perfusion defects in the lesion, after contrast agent administration; (d) lesion's enhancement order. Centripetal enhancement was defined as enhancement originating from the lesion's periphery towards its center, while centrifugal enhancement begins from the lesion's center, developing towards its periphery; (e) presence or absence of radial-penetrating vessels from surrounding tissue towards the lesion; (f) blurring lesion margins. Lesion margins were characterized as clear when most of its circumference was clearly visible $(>50 \%)$ and blurred when $>50 \%$ of its circumference was poorly visible after contrast agent administration; (g) presence or absence of perilesional enhancement; (h) internal sentinel lymph node homogeneity, characterized as homogenous when enhancement was visible and equal to whole lymph node cortex and heterogeneous when unenhanced areas or areas of different enhancement degree appeared after contrast medium administration.

On the other hand, quantitative parameters, resulted from the timeintensity curve (TIC), included the following: (a) peak intensity (\%) 
(PEAK), defined as maximum intensity value in time-intensity curve, calculated by the fraction

[(post-contrast intensity - pre-contrast intensity)/pre-contrast intensity] $\times 100 \%$;

(b) time to peak (sec) (TTP), defined as the time elapsed between the moment when contrast medium first reaches the lesion and the time of maximum signal intensity after contrast medium administration; (c) mean transit time (sec) (MTT), defined as the lesion's enhancement duration; (d) regional blood volume (ml) (RBV), which is the area under time-intensity curve, reflecting total volume of contrast medium (or blood) traversing the region of interest; (e) regional blood flow $(\mathrm{ml} / \mathrm{sec})(\mathrm{RBF})$, calculated by the fraction area under the curve/mean transit time, which reflects relative blood flow in the selected lesion's area. All these quantitative parameters are schematically presented in Figure 1.

The time-intensity curve was a result of the kinetic evaluation of a lesion's vascularization. All video recordings were analyzed offline in Matlab ${ }^{\circledR}$ R2013a (The MathWorks Inc., Natick, MA, USA). Specifically, a region of interest was manually positioned in the area most perfused within each lesion and median signal intensity (SI) was calculated over time on the frame-by-frame basis. Selected regions of interest size were about $16 \mathrm{~mm}^{2}$, depending on the lesion's extent. In cases where respiratory motion was high, several consecutive regions of interest were selected, in order to follow the initially selected area and thus improve the accuracy of quantification in perfusion evaluation. Videos where this correction was not possible were excluded from the study, as already referred to in the exclusion criteria On the resulting TIC, a corrected gamma-variate model (22) was fitted as defined in quantitative perfusion analysis software Qontrast ${ }^{\circledR}$ (Bracco, Milan, Italy) such as

$$
S I(t)=S I_{B L}+P E A K \times(t / T T P)^{(\beta \times T T P)} \times \exp ^{-\beta \times(t-T T P)}
$$

where SI is signal intensity extracted from the image during contrast agent bolus passage, $\mathrm{SI}_{\mathrm{BL}}$ is signal intensity baseline, which allows brightness correction when darkness is not complete at time zero, PEAK is maximum SI reached during the bolus transit of the contrast agent, at time t equals to TTP and $\beta$ is the steepness of bolus transit. An indicative example is depicted in Figure 2.

Pathological and immunohistochemical analysis. All patients underwent surgical resection of their lesions, 1-2 days after contrast enhanced ultrasound examination. A pathological verification of excised specimens with immunohistochemical analysis was performed by two pathologists, experienced in breast pathological analysis. Pathological diagnosis was considered as gold standard.

Tumors' histological typing was based on WHO classification (23). Tumor grade was assessed according to Elston-Ellis grading system (24). Axillary lymph nodes were assessed histologically on routinely stained sections and metastatic involvement of any size gave them a positive diagnosis, concerning their infiltration.

Immunohistochemical analysis includes the following pathological prognostic factors: (a) estrogen receptor (ER). Cut-off point for ER positivity was $\geq 10 \%(25,26)$; (b) progesterone receptor (PR). Cut-off point for PR positivity was $\geq 10 \%$; (c) Ki-67 gene expression, assessed by determining the percentage of positively stained tumor nuclei out of all tumor cells, with positivity threshold of $>20 \%$ of stained cells $(17,27)$; (d) p53 gene expression, considered positive in cases where well-defined nuclear staining was observed in more than $10 \%$ of tumor cells; (e) c-erb-B2 gene expression, defined as positive by revealing

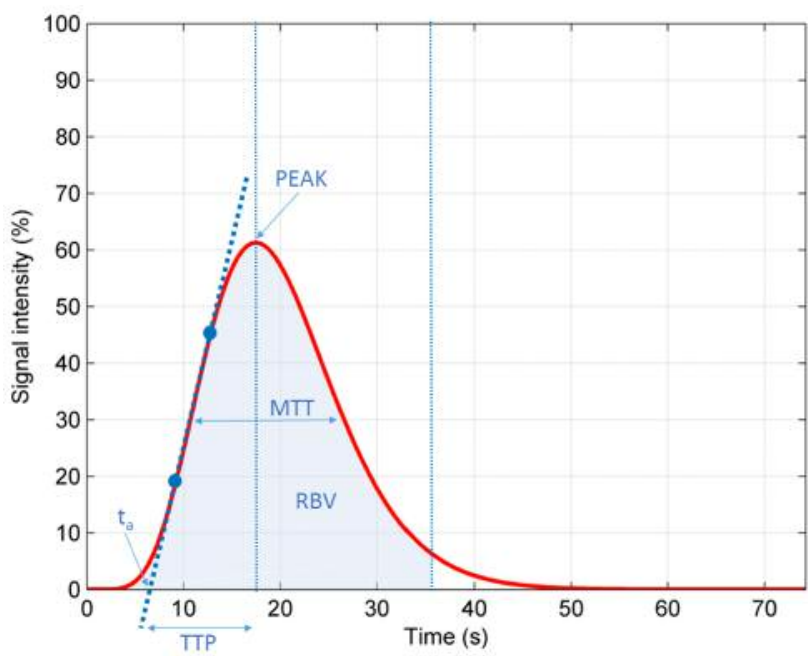

Figure 1. Graphic representation of time-intensity curve (TIC) and resulting CEUS quantitative parameters. PEAK: Peak intensity (\%); TTP: time to peak; MTT: mean transit time; RBV: regional blood volume; ta: time elapsed between the contrast agent injection and its arrival into the lesion.

moderate to high distinctive membranous staining in almost all tumor cells, while negativity was expressed by negative to low membrane straining in more than $10 \%$ of tumor cells (17).

Ethical issues. The present study was conducted in accordance to the declaration of Helsinki and it was approved by our institution's research ethics committee. All patients were informed of the study's purpose and methods and they gave their written informed consent. At the same time, the ultrasound department, where procedure took place, was adequately equipped, in order to encounter any side effects that may have appeared during intravenous contrast agent injection. No side effects were noted during the entire study.

Statistical analysis. Categorical variables are expressed as numbers (percentages), while continuous variables are expressed as mean, standard deviation, median, range, minimum value and maximum value. The Shapiro-Wilk criterion ( $p>0.05$ for all variables) and graphs (histograms and normal Q-Q plots) were used to test the normality of distribution of continuous variables. PEAK, TTP and RBF followed normal distribution, while RBV and MTT did not follow normal distribution.

Presence of ER and PR was transformed into dichotomous variables according to the following way: absence of receptors and low positive receptors categorized as absence of receptors, while intermediate and highly positive receptors categorized as presence of receptors. Similarly, c-erb-B2 and p53 genes' expression were transformed into dichotomous variables according to the following way: no c-erb-B2 or p53 genes' expression and low c-erb-B2 and p53 genes' expression identified as negative c-erb-B2 or p53 genes' expression, while intermediate and highly positive c-erb-B2 and p53 genes' expression identified as positive c-erb-B2 and p53 genes' expression. Furthermore, Ki-67 proliferation index transformed into dichotomous variable according to the following model: low and intermediate Ki-67 proliferation index considered as negative Ki-67 

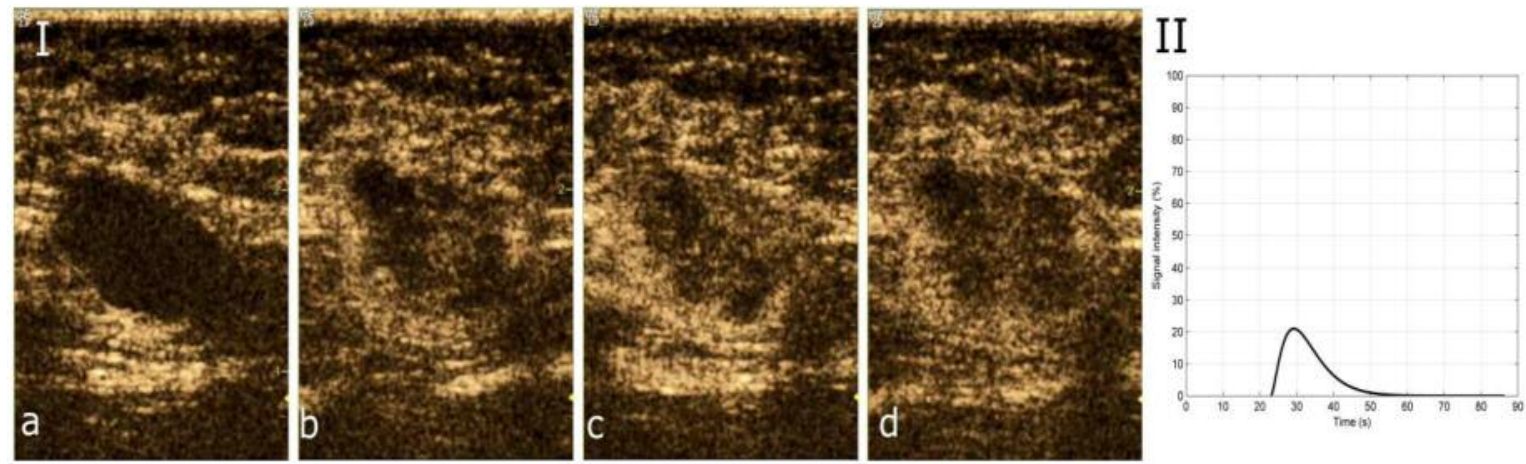

Figure 2. Characteristic images of invasive breast carcinoma from CEUS video and resulting graphic representation of time-intensity curve (TIC) of the lesion. (I) Four characteristic images from CEUS video in an invasive breast carcinoma, the moment (a) prior to contrast agent arrival ( $t=0$ sec), (b) of wash-in ( $t=25 \mathrm{sec}$ ), (c) of contrast agent PEAK intensity ( $t=31 \mathrm{sec}$ ), (d) of wash-out ( $t=48 \mathrm{sec}$ ). (II) The resulting TIC, corresponding to the lesion.

proliferation index, while high $\mathrm{Ki}-67$ proliferation index considered as positive Ki-67 proliferation index. Likewise, cut-off point of $2 \mathrm{~cm}$ was used in order to dichotomize lesions' diameter. Two qualitative characteristics, heterogeneous enhancement and enhancement order have not been used for correlations, due to limited variability in some categories $(\mathrm{n} \leq 1)$.

Pathological prognostic factors were dependent variables, while CEUS characteristics were independent variables. Bivariate analyses between CEUS characteristics and pathological prognostic factors included chi-square test and Fisher's exact test for categorical variables and student's $t$-test and Mann-Whitney test for continuous variables. The independent variables which were significantly different $(p<0.2)$ in bivariate analyses were entered into the backward stepwise multivariate logistic regression analysis with pathological prognostic factors as dependent variables. Adjusted odds ratios (OR) with $95 \%$ confidence intervals (CI) and $p$-values were estimated for the independent variables included in final models.

Bonferroni correction was not applied due to the exploratory nature of the study and to the relatively small sample size. All tests of statistical significance were two-tailed and $p$-values $<0.05$ were considered significant. Statistical analysis was performed with the IBM SPSS 21.0 (IBM Corp. IBM SPSS Statistics, Version 21.0. Armonk, NY: IBM Corp.).

\section{Results}

Histopathological and immunohistochemical analysis. All malignant breast lesions $(n=34)$ were primary breast cancers and included invasive carcinoma of no special type $(n=21,61.7 \%)$, invasive carcinoma of no special type and invasive lobular carcinoma $(n=4,11.8 \%)$, invasive carcinoma of no special type and invasive mucinous carcinoma $(n=3,8.8 \%)$, invasive carcinoma of no special type and cribriform carcinoma $(n=1$, $2.9 \%$ ), invasive lobular carcinoma $(n=1,2.9 \%)$, invasive papillary carcinoma $(\mathrm{n}=1,2.9 \%)$, invasive micropapillary carcinoma $(n=1,2.9 \%)$, invasive papillary and mucinous carcinoma $(\mathrm{n}=1,2.9 \%)$, and invasive carcinoma with medullary features $(\mathrm{n}=1,2.9 \%)$. Tumor's size ranged from 0.7 to $8 \mathrm{~cm}$, mean size was $2.4 \mathrm{~cm}$ (standard deviation; 1.4) and median size
Table I. Quantitative CEUS characteristics of the 34 malignant lesions.

\begin{tabular}{lrrrrr}
\hline Parameter & Mean & $\begin{array}{c}\text { Standard } \\
\text { deviation }\end{array}$ & Median & $\begin{array}{c}\text { Minimum } \\
\text { value }\end{array}$ & $\begin{array}{c}\text { Maximum } \\
\text { value }\end{array}$ \\
\hline PEAK (\%) & 55.12 & 22.21 & 50.25 & 21.00 & 92.64 \\
TTP $(\mathrm{sec})$ & 15.27 & 7.09 & 13.84 & 5.90 & 38.59 \\
MTT $(\mathrm{sec})$ & 21.89 & 11.56 & 19.22 & 8.18 & 57.74 \\
RBV $(\mathrm{ml})$ & 1336.71 & 991.03 & 934.96 & 270.04 & 4040.44 \\
RBF $(\mathrm{ml} / \mathrm{sec})$ & 58.89 & 23.44 & 54.44 & 22.53 & 101.80 \\
\hline
\end{tabular}

PEAK: Peak intensity (\%); TTP: time to peak; MTT: mean transit time; RBV: regional blood volume; RBF: regional blood flow.

was $2.2 \mathrm{~cm}$. Of all 34 malignant breast lesions, 22 lesions $(64.7 \%)$ were $\geq 2 \mathrm{~cm}$ in diameter, 13 lesions (38.2\%) were poorly differentiated (grade III), 19 lesions (55.9\%) were intermediately differentiated (grade II) and two lesions (5.9\%) were highly differentiated (grade I). Sentinel lymph node infiltration was present in 13 cases (38.2\%), while lesion necrosis was present in 8 cases $(23.5 \%)$. Among 33 malignant breast lesions, there were $24(72.8 \%)$ ER-positive lesions, 13 (39.4\%) PR-positive lesions, 17 (51.5\%) c-erb-B2 positive lesions and $15(45.5 \%) \mathrm{Ki}-$ 67 positive lesions, while among 31 malignant breast lesions, there were 7 (22.6\%) p53-positive lesions.

CEUS analysis. Of the 34 malignant breast lesions, 16 (47.1\%) were characterized as hypoenhanced compared to surrounding tissue, $11(32.3 \%)$ were characterized as isoenhanced compared to surrounding tissue and $7(20.6 \%)$ did not show any enhancement. All 27 enhancing malignant breast lesions showed centripetal enhancement $(100 \%)$, while enhancement was heterogeneous in 26 of them $(96.3 \%)$. Radial vessels were imaged in 25 lesions $(73.5 \%)$ and at least one perfusion defect was observed in 26 lesions $(76.5 \%)$, while perilesional enhancement was demonstrated in 26 lesions (76.5\%). Among 
31 malignant breast lesions, blurred lesion margins were observed in 20 lesions (64.5\%). Last, heterogeneous sentinel lymph node enhancement was depicted in 15 lesions (44.1\%).

Descriptive statistics of CEUS quantitative characteristics (PEAK, TTP, MTT, RBV and RBF) of 34 malignant lesions are shown in Table I.

Association between CEUS characteristics and pathological prognostic factors. Bivariate analyses between qualitative and quantitative CEUS characteristics and pathological prognostic factors are shown in Tables II and III.

Referring to qualitative CEUS characteristics, lesion's isoenhancement to surrounding tissue and internal sentinel lymph node heterogeneity were seen in tumors with sentinel lymph node infiltration ( $p=0.006$ and $p<0.001$ respectively). Blurred lesion margins and perilesional enhancement were common in lesions with higher histologic grade $(p=0.001$ and $p=0.03$ respectively). In addition, blurred lesion margins were observed in ER-negative tumors $(p=0.01)$, while perilesional enhancement was seen in Ki-67-positive tumors $(p=0.049)$.

With regards to quantitative CEUS characteristics, median MTT was lower in larger tumors (diameter $\geq 2 \mathrm{~cm})(p=0.04)$.

Logistic regression analysis. Logistic regression models with statistically significant relationships are summarized in Table IV. Blurred lesion margins were found more often in tumors with higher histologic grade and negative ER expression. In addition, perilesional enhancement was associated with positive Ki-67 expression, while heterogeneous sentinel lymph node enhancement was associated with sentinel lymph node infiltration.

\section{Discussion}

Angiogenesis is a fundamental process in neoplastic tumor growth. Therefore, identifying key pathways involved in this process could lead to more effective and targeted therapeutic managements of oncology patients $(28,29)$. Modern imaging methods, like CEUS, could provide a non-invasive method of evaluation of a tumor's angiogenesis. CEUS reflects angiogenesis better than contrast-enhanced magnetic resonance imaging (MRI), since contrast agent used in CEUS is a real blood-pool agent, without diffusion $(17,30)$. Moreover, there are studies which have already shown that CEUS can discriminate malignant from benign breast lesions (31-34), since malignant breast lesions more often demonstrate heterogeneous and centripetal enhancement, while benign lesions demonstrate homogeneous and centrifugal enhancement. Concerning quantitative CEUS characteristics, malignant lesions show faster and higher enhancement than benign ones. Therefore, this sophisticated ultrasound imaging technique, could probably contribute enough in breast cancer diagnosis and prognosis.
In our study, multivariate analyses were performed and statistically significant relations were found between some of the qualitative CEUS characteristics (blurring lesion margins, perilesional enhancement and heterogeneous enhancement of the sentinel lymph node) and pathological prognostic factors, which indicate tumor aggressiveness (ER expression, histologic grade, Ki-67 expression and sentinel lymph node infiltration). These relations suggest that CEUS could play a significant role in breast cancer prognosis. Our results revealed that blurred lesion margins were the most important CEUS characteristic in prognosis of breast cancer, since it was the only characteristic related to two pathological prognostic factors; ER expression and histologic grade.

Blurred lesion margins after the contrast agent administration are a characteristic indicating malignancy in all imaging methods (35). In CEUS, blurring lesion margins after contrast agent administration is attributed to an ongoing angiogenic procedure, corresponding to tumor's malignant activity and expansion in the area. This imaging finding is compatible to a higher histologic grade, which is one of the best-established independent pathological prognostic factors in breast cancer, with higher grade indicating worse prognosis (36). This was the first correlation found in this study, in which blurred lesion margins appeared more often in lesions with a higher histologic grade (grade III). All the same, histologic grade has already been correlated with other qualitative CEUS characteristics in literature. To illustrate, Colpaert et al. (37) found that tumors of higher histologic grade are more susceptible to central necrosis than tumors of lower histologic grade, while other researchers correlated penetrating vessels and perfusion defects with grade III tumors $(14,15,17)$.

In our study, blurring lesion margins were also more often observed in ER negative tumors. It is probable that angiogenic factors produced by more aggressive ER negative tumors, along with rapid growth of these tumors, result in blurred lesion margins in CEUS. Absence of ER expression has already been related to other qualitative and quantitative CEUS characteristics in literature, like more common perfusion defects $(14,15,17)$ or centripetal enhancement (15), as well as higher PEAK intensity (13). To illustrate, perfusion defects are the result of rapid growth and active metabolism of malignant tumors, leading to insufficient vascular formation and nutrition supply and finally to central hypoxia and necrosis (38). Moreover, Liu et al. attribute perfusion defects to low cellularity, desmoplastic stroma, dilated duct, degeneration, fibrosis or necrosis (18). According to the literature, central necrosis and fibrosis are common in ER negative tumors $(39,40)$, while regional hypoxia is associated with reduced ER expression in breast cancer patients (41). On the other hand, ER positive patients have a better prognosis (42), unlike breast cancers characterized by centripetal enhancement, which do not respond well to neo-adjuvant chemotherapy (43). Therefore, centripetal enhancement and perfusion defects should be more common in ER negative tumors. 


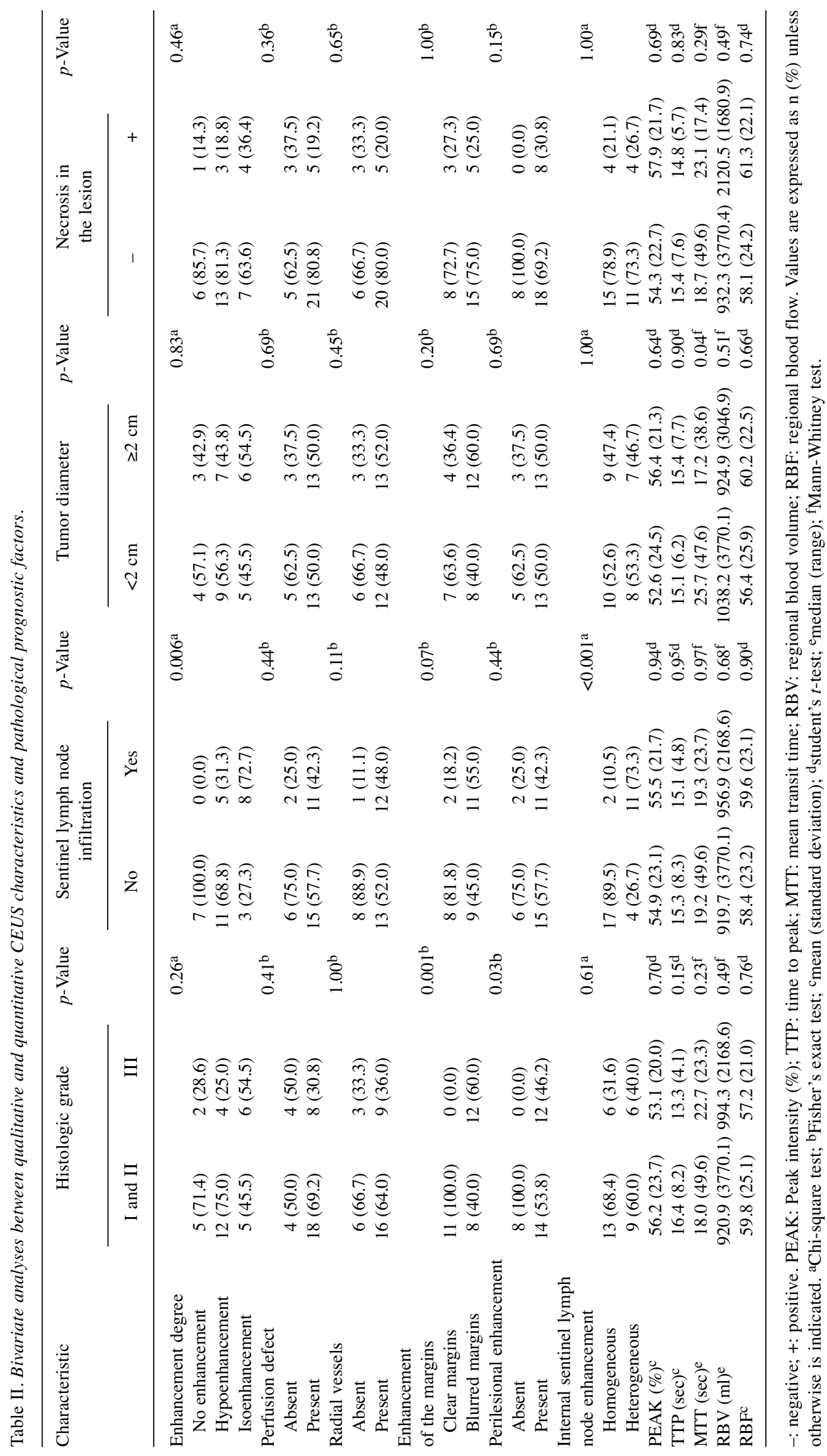




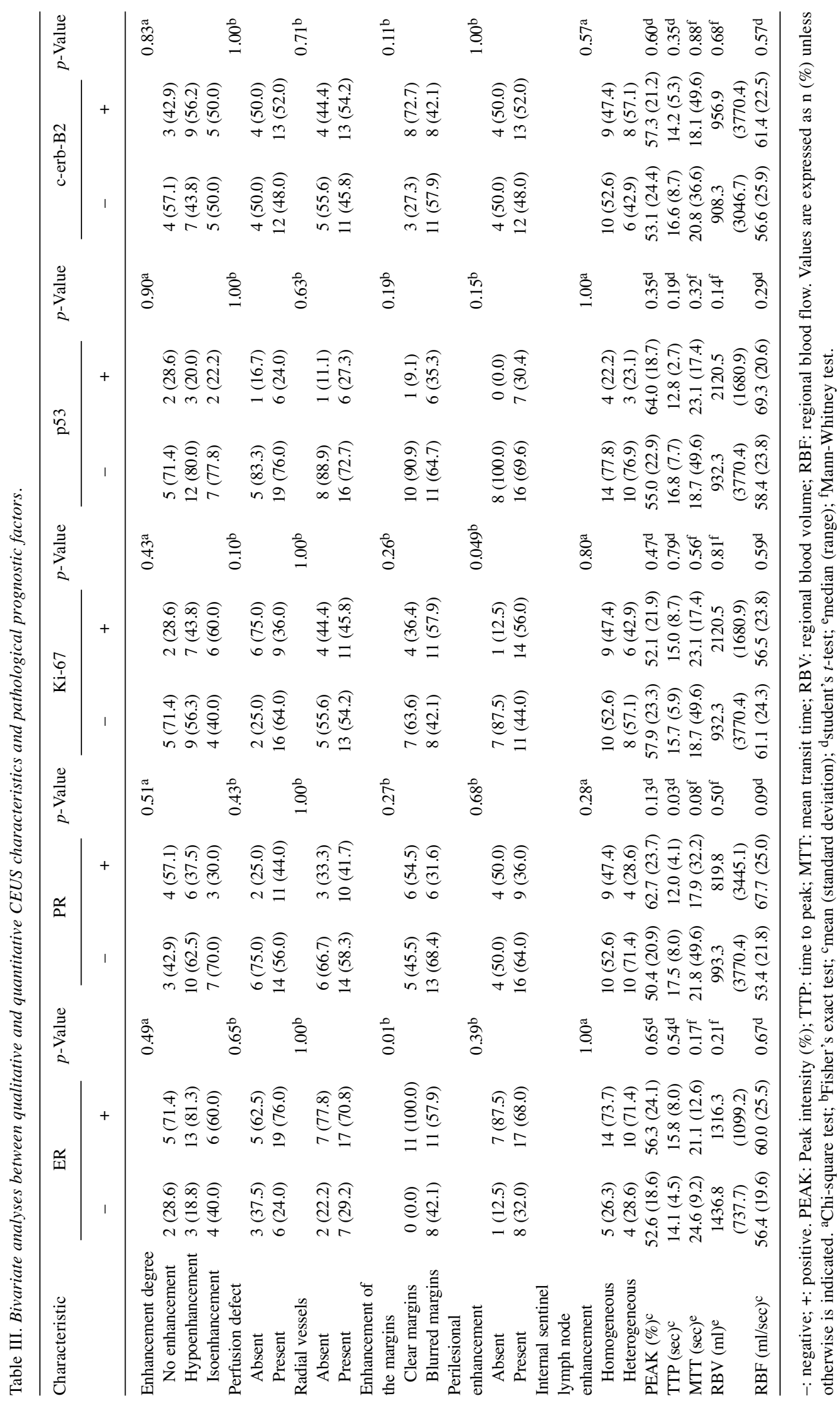


Table IV. Logistic regression analyses with pathological prognostic factors as dependent variables and CEUS characteristics as independent variables.

\begin{tabular}{lcc}
\hline $\begin{array}{l}\text { Dependent variable } \\
\text { Independent variable }\end{array}$ & Odds ratio & $95 \%$ confidence interval \\
\hline $\begin{array}{l}\text { Histologic grade (III } v s . \text { I and II) } \\
\quad \text { Blurred lesion margins compared to clear }\end{array}$ \\
$\begin{array}{l}\text { Sentinel lymph node infiltration (yes } v s . \text { no) } \\
\quad \begin{array}{l}\text { Heterogeneous internal enhancement of sentinel lymph node } \\
\text { compared to homogeneous internal enhancement }\end{array}\end{array}$ & 8.08 & 1.64 to 39.75 \\
$\begin{array}{l}\text { ER (positive } v s . \text { negative) } \\
\quad \text { Clear lesion margins compared to blurred lesion margins }\end{array}$ & 45.76 & 3.91 to 535.07 \\
$\begin{array}{l}\text { Ki-67 (positive } v s . \text { negative) } \\
\quad \text { Perilesional enhancement compared to absence of perilesional enhancement }\end{array}$ & 9.00 & 1.00 to 82.49 \\
\hline
\end{tabular}

As already mentioned, $\mathrm{Ki}-67$ is an independent prognostic molecular biomarker in breast cancer, with well-established reliability. Positive Ki-67 expression corresponds to increased proliferation activity and poor prognosis $(9,44)$. Zhao et al. (14) and Cao et al. (15) found that positive Ki-67 expression was correlated with perfusion defects in tumors, while Wan et al. (17) argued that the ratio of the number of lesions with high microvessel density to the number of lesions with low microvessel density was higher in tumors with positive Ki-67 expression. In our study, positive Ki-67 expression correlated with perilesional enhancement. This finding implies that angiogenic factors produced by tumors with high proliferative activity, may exert significant effects on the surrounding tissue, provoking local angiogenesis and thus perilesional enhancement $(45,46)$.

Finally, in the present study, heterogeneous sentinel lymph node enhancement was correlated with its pathologically proven infiltration. This finding is supported by previous research, suggesting that heterogeneous enhancement reflects differences in microvessel density and distribution of vasculature (47). All the same, heterogeneity has already been correlated to malignant potential of breast lesions $(14,31,33)$ and some markers of poor prognosis, such as tumor diameter $>2 \mathrm{~cm}$ and positive c-erb-B2 expression $(14,15,17)$. Furthermore, rapid growth and active metabolism of malignant tumors lead to insufficient vascular formation and nutrition supply and then to local ischemic necrosis (38). These findings could explain and support our observation.

As opposed to qualitative CEUS characteristics, no correlation between quantitative CEUS characteristics and pathological prognostic factors was observed. Further investigation in this area could provide an objective tool for the evaluation of breast tumors, which would be, free from the subjectivity characterizing the qualitative study with CEUS. It is probable that in qualitative CEUS parameters, interpreter's experience corrects possible methodological faults, a correction which cannot be done in quantitative parameters. Namely, small patient's movements, due to respiration or not, during video recording, do not significantly affect the qualitative evaluation, while being important for the placement of the region of interest on the received images, during the quantitative evaluation. In our study, we were obliged to separate some patients' video in two or more parts during the quantitative study, each one of which had a different place of the region of interest on the received images, in an effort to make our measures at the very same region of the lesion, limiting in this way the resulting methodological fault, which apparently affects the quantitative study's results. In addition, present quantitative CEUS parameters are based on quantitative MRI parameters, even though CEUS and MRI contrast agents reflect different tissue distribution, as already mentioned. Moreover, MRI gives the interpreter the option to select the region of interest from the whole area of a lesion, whereas CEUS provides only a preselected area by the CEUS performer. A potential answer to this methodological restriction could be the receipt of two different measurements on two different regions of interest, in two different sections of the lesion, after the administration of the contrast agent twice. Alternatively, we could have two or more measurements of different places on the same image. Thus, further studies on this domain are truly reasonable, taking into account and trying to overcome all these restrictions, as CEUS have the potential to provide an objective preoperative diagnostic tool. The strengths of our study are worth mentioning and include (a) investigation both of qualitative and quantitative CEUS characteristics, (b) investigation of a great number of pathological prognostic factors in breast cancer patients and (c) use of multivariate analysis, in order to eliminate confounding. To our knowledge, only Wan et al. (17) investigated both qualitative and quantitative CEUS characteristics, performing multivariate analysis.

Besides, an advantage worth mentioning is that no allergic reactions were noted in any of our patients and this is a known advantage of microbubble contrast agents, according to the 
literature (48). On the other hand, gadolinium-based contrast agents used in MRI examinations have a small, but welldocumented risk for hypersensitivity reactions, as well as the recent reports of gadolinium deposition in the brain, after repeated administration of the contrast agent (49).

However, our study had also some limitations. Firstly, sample size was relatively small and consequently random error was high, as indicated by wide confidence intervals for odds ratios in regression models. Therefore, further studies, with larger sample size are needed. Secondly, enhancement pattern classification for the qualitative lesions' study and region of interest selection for the quantitative study were subjective. Thirdly, the study included only malignant tumors. Thus, comparison between malignant and benign breast lesions was not carried out, as it was beyond the purpose of our study. Future studies should evaluate differences in terms of qualitative and the quantitative CEUS characteristics between malignant and benign breast lesions. Finally, we performed a single-center study and selection bias was unavoidable. Future multi-center studies could also overcome this limitation.

In conclusion, the present study confirmed that some CEUS characteristics are related with established pathological prognostic factors in breast cancer, indicating that CEUS has the potential to be a useful, non-invasive and inexpensive method compared to the established diagnostic procedures in the domain of breast cancer. Finally, our results could be beneficial in the future, in the effort to find clinical applications of CEUS in breast cancer prognosis.

\section{References}

1 Rojas K and Stuckey A: Breast cancer epidemiology and risk factors. Clin Obstet Gynecol 59: 651-672, 2016.

2 Tao Z, Shi A, Lu C, Song T, Zhang Z and Zhao J: Breast cancer: epidemiology and etiology. Cell Biochem Biophys 72: 333-338, 2015.

3 Ban KA and Godellas CV: Epidemiology of breast cancer. Surg Oncol Clin N Am 23: 409-422, 2014.

4 Soerjomataram I, Louwman MW, Ribot JG, Roukema JA and Coebergh JW: An overview of prognostic factors for long-term survivors of breast cancer. Breast Cancer Res Treat 107: 309330,2008

5 Diaz LK and Sneige N: Estrogen receptor analysis for breast cancer: current issues and keys to increasing testing accuracy. Adv Anat Pathol 12: 10-19, 2005.

6 Gown AM: Current issues in ER and HER2 testing by IHC in breast cancer. Mod Pathol 21(Suppl 2): S8-S15, 2008.

7 American Society of Clinical Oncology: Clinical practice guidelines for the use of tumor markers in breast and colorectal cancer. Adopted on May 17, 1996 by the American Society of Clinical Oncology. J Clin Oncol 14: 2843-2877, 1996.

8 Guarneri V, Broglio K, Kau SW, Cristofanilli M, Buzdar AU, Valero V, Buchholz, T, Meric F, Middleton L, Hortobagyi GN and Gonzalez-Angulo AM: Prognostic value of pathologic complete response after primary chemotherapy in relation to hormone receptor status and other factors. Clin Oncol 24: 1037-1044, 2006.
9 Kutomi G, Mizuguchi T, Satomi F, Maeda H, Shima H, Kimura $\mathrm{Y}$ and Hirata K: Current status of the prognostic molecular biomarkers in breast cancer: A systematic review. Oncol Lett 13: 1491-1498, 2017.

10 Li BJ, Zhu ZH, Wang JY, Hou JH, Zhao JM, Zhang PY, Yao GY, Wang X, Long H, Yang MT and Rong TH: Expression correlation of Ki67 to P53, VEGF, and C-erbB-2 genes in breast cancer and their clinical significances. Ai Zheng 23: 1176-1179, 2004.

11 Beenken SW, Grizzle WE, Crowe DR, Conner MG, Weiss HL, Sellers MT, Krontiras H, Urist MM and Bland KI: Molecular biomarkers for breast cancer prognosis: coexpression of c-erbB2 and p53. Ann Surg 233: 630-638, 2001.

12 de Azambuja E, Cardoso F, de Castro G Jr, Colozza M, Mano MS, Durbecq V, Sotiriou C, Larsimont D, Piccart-Gebhart MJ and Paesmans M: Ki-67 as prognostic marker in early breast cancer: a meta-analysis of published studies involving 12,155 patients. Br J Cancer 96: 1504-1513, 2007.

13 Ji CL, Li XL, He YP, Li DD, Gu XG and Xu HX: Quantitative parameters of contrast-enhanced ultrasound in breast invasive ductal carcinoma: The correlation with pathological prognostic factors. Clin Hemorheol Microcirc 66: 333-345, 2017.

14 Zhao YX, Liu S, Hu YB, Ge YY and Lv DM: Diagnostic and prognostic values of contrast-enhancement ultrasound in breast cancer: a retrospective study. Onco Targets Ther 10: 1123-1129, 2017.

15 Cao XL, Bao W, Zhu SG, Wang LH, Sun MH, Wang L, Men YM and Xue J: Contrast-enhanced ultrasound characteristics of breast cancer: correlation with prognostic factors. Ultrasound Med Biol 40: 11-17, 2014.

16 Szabó BK, Saracco A, Tánczos E, Aspelin P, Leifland K, Wilczek B and Axelsson R: Correlation of contrast-enhanced ultrasound kinetics with prognostic factors in invasive breast cancer. Eur Radiol 23: 3228-3236, 2013.

17 Wan C, Du J, Fang H, Li F, Zhu JS and Liu Q: Enhancement patterns and parameters of breast cancers at contrast-enhanced US: correlation with prognostic factors. Radiology 262: 450-459, 2012.

18 Zhao LX, Liu H, Wei Q, Xu G, Wu J, Xu HX, Wu R and Pu H: Contrast-enhanced ultrasonography features of breast malignancies with different sizes: correlation with prognostic factors. Biomed Res Int ID: 613831, 2015.

19 Wang XY, Hu Q, Fang MY, He Y, Wei HM, Chen XX and Zhang B: The correlation between HER-2 expression and the CEUS and ARFI characteristics of breast cancer. PLoS One 12: e0178692, 2017.

20 Krishnamurthy S, Sneige N, Bedi DG, Edieken BS, Fornage BD, Kuerer HM, Singletary SE and Hunt KK: Role of ultrasoundguided fine-needle aspiration of indeterminate and suspicious axillary lymph nodes in the initial staging of breast carcinoma. Cancer 95: 982-988, 2002.

21 Albrecht T, Blomley M, Bolondi L, Claudon M, Correas JM, Cosgrove D, Greiner L, Jäger K, Jong ND, Leen E, Lencioni R, Lindsell D, Martegani A, Solbiati L, Thorelius L, Tranquart F, Weskott HP and Whittingham T; EFSUMB Study Group: Guidelines for the use of contrast agents in ultrasound. January 2004. Ultraschall Med 25: 249-256, 2004.

22 Thijssen JM and de Korte CL: Modeling ultrasound contrast measurement of blood flow and perfusion in biological tissue. Ultrasound Med Biol 31: 279-285, 2005.

23 Sinn HP and Kreipe H: A brief overview of the WHO classification of breast tumors, 4th Edition, Focusing on issues and updates from the 3rd Edition. Breast Care (Basel) 8: 149$154,2013$. 
24 Elston CW and Ellis IO: Pathological prognostic factors in breast cancer. I. The value of histological grade in breast cancer: experience from a large study with long-term follow up. Histopathology 19: 403-410, 1991.

25 Yi M, Huo L, Koenig KB, Mittendorf EA, Meric-Bernstam F, Kuerer HM, Bedrosian I, Buzdar AU, Symmans WF, Crow JR, Bender M, Shah RR, Hortobagyi GN and Hunt KK: Which threshold for ER positivity? A retrospective study based on 9639 patients. Ann Oncol 25: 1004-1011, 2014.

26 Iwamoto T, Booser D, Valero V, Murray JL, Koenig K, Esteva FJ, Ueno NT, Zhang J, Shi W, Qi Y, Matsuoka J, Yang EJ, Hortobagyi GN, Hatzis C, Symmans WF and Pusztai L: Estrogen receptor (ER) mRNA and ER-related gene expression in breast cancers that are $1 \%$ to $10 \%$ ER-positive by immunohistochemistry. J Clin Oncol 30: 729-734, 2012.

27 Penault-Llorca F, André F, Sagan C, Lacroix-Triki M, Denoux Y, Verriele V, Jacquemier J, Baranzelli MC, Bibeau F, Antoine M, Lagarde N, Martin AL, Asselain B and Roché H: Ki67 expression and docetaxel efficacy in patients with estrogen receptor-positive breast cancer. J Clin Oncol 27: 2809-2815, 2009.

28 Orgaz JL, Martínez-Poveda B, Fernández-García NI and Jiménez B: Following up tumour angiogenesis: from the basic laboratory to the clinic. Clin Transl Oncol 10: 468-477, 2008.

29 Bridges EM and Harris AL: The angiogenic process as a therapeutic target in cancer. Biochem Pharmacol 81: 1183-1191, 2011.

30 Caproni N, Marchisio F, Pecchi A, Canossi B, Battista R, D'Alimonte P and Torricelli P: Contrast-enhanced ultrasound in the characterisation of breast masses: utility of quantitative analysis in comparison with MRI. Eur Radiol 20: 1384-1395, 2010.

31 Balleyguier C, Opolon P, Mathieu MC, Athanasiou A, Garbay JR, Delaloge S and Dromain C: New potential and applications of contrast-enhanced ultrasound of the breast: own investigations and review of the literature. Eur J Radiol 69: 14-23, 2009.

32 Zhao H, Xu R, Ouyang Q, Chen L, Dong B and Huihua Y: Contrast-enhanced ultrasound is helpful in the differentiation of malignant and benign breast lesions. Eur J Radiol 73: 288-293, 2010.

33 Wan C, Du J, Fang H, Li F and Wang L: Evaluation of breast lesions by contrast enhanced ultrasound: Qualitative and quantitative analysis. Eur J Radiol 81: e444-e450, 2012.

34 Blohmer JU, Reinhardt M, Schmalisch G, Lichtenegger W, Hausschild M, Kümmel S, Fischer T, Ohlinger R and Thomas A: Videodensitometry in the examination of focal breast lesions after injection of an ultrasound contrast agent. Anticancer Res 26: 1691-1698, 2006.

35 Fischer DR, Baltzer P, Malich A, Wurdinger S, Freesmeyer MG, Marx C and Kaiser WA: Is the "blooming sign" a promising additional tool to determine malignancy in MR mammography? Eur Radiol 14: 394-401, 2004.

36 Rakha EA, Reis-Filho JS, Baehner F, Dabbs DJ, Decker T, Eusebi V Fox SB, Ichihara S, Jacquemier J, Lakhani SR, Palacios J, Richardson AL, Schnitt SJ, Schmitt FC, Tan PH, Tse GM, Badve $S$ and Ellis IO: Breast cancer prognostic classification in the molecular era: the role of histological grade. Breast Cancer Res 12: 207, 2010.

37 Colpaert C, Vermeulen P, van Beest P, Goovaerts G, Weyler J, Van Dam P, Dirix L and Van Marck E: Intratumoral hypoxia resulting in the presence of a fibrotic focus is an independent predictor of early distant relapse in lymph node-negative breast cancer patients. Histopathology 39: 416-425, 2001.
38 Metz S, Daldrup-Unk HE, Richter T, Rath C, Ebert W, Settles M, Rummeny EJ, Link TM and Piert M: Detection and quantification of breast tumor necrosis with MR imaging: value of the necrosis-avid contrast agent Gadophrin-3. Acad Radiol 10: 484-490, 2003.

39 Putti TC, El-Rehim DM, Rakha EA, Paish CE, Lee AH, Pinder SE and Ellis I: Estrogen receptor-negative breast carcinomas: a review of morphology and immunophenotypical analysis. Mod Pathol 18: 26-35, 2005.

40 Iwase H, Ando Y, Ichihara S, Toyoshima S, Nakamura T, Karamatsu S, Ito Y, Yamashita H, Toyama T, Omoto Y, Fujii Y, Mitsuyama $S$ and Kobayashi S: Immunohistochemical analysis on biological markers in ductal carcinoma in situ of the breast. Breast Cancer 8: 98-104, 2001.

41 Bos R, van Diest PJ, van der Groep P, Shvarts A, Greijer AE and van der Wall E: Expression of hypoxia-inducible factor-1alpha and cell cycle proteins in invasive breast cancer are estrogen receptor related. Breast Cancer Res 6: R450-459, 2004.

42 Early Breast Cancer Trialists' Collaborative Group (EBCTCG), Davies C, Godwin J, Gray R, Clarke M, Cutter D, Darby S, McGale P, Pan HC, Taylor C, Wang YC, Dowsett M, Ingle J and Peto R: Relevance of breast cancer hormone receptors and other factors to the efficacy of adjuvant tamoxifen: patient-level metaanalysis of randomised trials. Lancet 378: 771-784, 2011.

43 Craciunescu OI, Blackwell KL, Jones EL, Macfall JR, Yu D, Vujaskovic Z, Wong TZ, Liotcheva V, Rosen EL, Prosnitz LR, Samulski TV and Dewhirst MW: DCE-MRI parameters have potential to predict response of locally advanced breast cancer patients to neoadjuvant chemotherapy and hyperthermia: A pilot study. Int J Hyperthermia 25: 405-415, 2009.

44 Thangarajah F, Enninga I, Malter W, Hamacher S, Markiefka B, Richters L, Krämer S, Mallmann $\mathrm{P}$ and Kirn V: A retrospective analysis of Ki-67 index and its prognostic significance in over 800 primary breast cancer cases. Anticancer Res 37: 1957-1964, 2017.

45 Lichtenbeld HC, Barendsz-Janson AF, van Essen H, Struijker Boudier H, Griffioen AW and Hillen HF: Angiogenic potential of malignant and non-malignant human breast tissues in an in vivo angiogenesis model. Int J Cancer 77: 455-459, 1998.

46 Brown LF, Berse B, Jackman RW, Tognazzi K, Guidi AJ, Dvorak HF, Senger DR, Connolly JL and Schnitt SJ: Expression of vascular permeability factor (vascular endothelial growth factor) and its receptors in breast cancer. Hum Pathol 26: 86-91, 1995.

47 Buadu LD, Murakami J, Murayama S, Hashiguchi N, Sakai S, Masuda K, Toyoshima S, Kuroki S and Ohno S: Breast lesions: correlation of contrast medium enhancement patterns on MR images with histopathologic findings and tumor angiogenesis. Radiology 200: 639-649, 1996.

48 Tang C, Fang K, Guo Y, Li R, Fan X, Chen P, Chen Z, Liu Q and Zou Y: Safety of sulfur hexafluoride microbubbles in sonography of abdominal and superficial organs: retrospective analysis of 30,222 cases. J Ultrasound Med 36: 531-538, 2017.

49 Rogosnitzky $\mathrm{M}$ and Branch S: Gadolinium-based contrast agent toxicity: A review of known and proposed mechanisms. BioMetals 29: 365-376, 2016.

Received March 22, 2018

Revised April 21, 2018

Accepted April 23, 2018 